\title{
ANÁLISE DOS RUÍDOS RESPIRATÓRIOS DE CAVALOS ATLETAS NO DIAGNÓSTICO DA HEMIPLEGIA DE LARINGE
}

\section{Analysis of respiratory sounds of performance horses in the diagnosis of laringeal hemiplegia}

DORNBUSCH, P.T. ${ }^{1}$; LEITE, S.C. ${ }^{1}$; CIRIO, S.M. ${ }^{1}$; PIMPÃO, C.T. ${ }^{1}$; LUNELLI, D. ${ }^{1}$; MICHELLOTTO JR, P.V. ${ }^{1}$; LEITE, L.C. ${ }^{2}$

${ }^{1}$ Pontifícia Universidade Católica do Paraná

${ }^{2}$ Unidade de Ensino Superior Vale do Iguaçu - UNIGUAÇU-PR

Endereço para correspondência: Peterson Triches Dornbusch - p.triches@pucpr.br

\section{RESUMO}

A hemiplegia de laringe é uma das principais afecções das vias aéreas superiores nos cavalos. Pesquisas de novos métodos para determinar o efeito dos diferentes graus de hemiplegia na performance atlética de cavalos têm sido realizadas. $O$ objetivo do presente estudo foi avaliar a eficiência da análise dos ruídos respiratórios no diagnóstico da hemiplegia de laringe, durante a atividade física intensa em condições reais de exercício em pista. Foram utilizados dez cavalos divididos em três grupos: animais saudáveis (controle), animais induzidos experimentalmente a hemiplegia de laringe (HLI), e animais naturalmente acometidos pela doença (HL). A gravação do áudio foi realizada através de sonda intranasal, posicionada na faringe, contendo microfone conectado a um gravador digital, cujos dados foram posteriormente transferidos para um computador e analisados. Os decibéis inspiratórios máximos dos Grupos HLI e HL demonstraram diferença significativa quando comparados ao grupo controle, e os demais dados avaliados não apresentaram diferenças significativas. A amplitude máxima dos decibéis inspiratórios mostrou ser significativamente diferente nos animais com hemiplegia de laringe natural e induzida, podendo desta forma ser utilizada no diagnóstico da interferência da hemiplegia de laringe no desempenho atlético de cavalos em condições de campo.

Palavras-chave: cartilagem aritenóide; vias aéreas superiores; decibéis inspiratórios.

\section{ABSTRACT}

Hemiplegia of the larynx is one of the main diseases that affect the upper airway in horses. Alternative methods have been researched to determine the effects of different degrees of hemiplegia on the performance of horses. The objective of this study was to evaluate the effectiveness of employing the analysis of respiratory sounds to diagnose hemiplegia of the larynx during intense physical activity while in real conditions exercising on the track. Ten horses were used to perform this study; the horses were divided into three groups: healthy animals (control group), animals that underwent an experimental induction of hemiplegia of the larynx (HLI), andanimals naturally affected by the disease $(\mathrm{HL})$. An intranasal probe containing a microphone attached to a digital recorder was positioned in the pharynx and used to record the sounds. The sounds were transferred to a laptop and analyzed. The maximum decibel of the inhalation of HLI and $\mathrm{HL}$ demonstrated a significant difference when compared with control group, while the other data showed no significant differences. The magnitude of the maximum decibels of inhalation were shown to be significantly different in animals with both natural and induced forms of hemiplegia of the larynx; this method can thus be used in the diagnosis of hemiplegia of the larynx and its interference with the athletic performance of horses in field conditions.

Key words: aritenoid cartilage, upper airway, inspiratory decibels. 


\section{INTRODUÇÃO}

Os maiores prejuízos econômicos em cavalos de esporte são conseqüentes a problemas locomotores, seguidos a problemas respiratórios. Dentre todas as afecções que acometem as vias aéreas superiores, a principal é a hemiplegia ou paralisia de laringe, doença idiopática que de acordo com Hammer et al. (1999) e Marti et al. (2002), pode ocorrer uni ou bilateralmente, sendo o lado esquerdo mais afetado. A hemiplegia de laringe resulta na não-abdução da cartilagem aritenóide durante a inspiração, levando a uma queda no fluxo do ar, aumento na resistência inspiratória e queda nas trocas gasosas em nível pulmonar (Holcombe, 2006; Morris e Seeherman, 1990). Como método diagnóstico, utiliza-se a endoscopia para avaliação e identificação desta enfermidade (Robertson e Ducharme, 2005). Porém, observa-se dificuldade neste tipo de avaliação com o animal em repouso, pois nem sempre há boa correlação com os achados endoscópicos, clínicos e de desempenho atlético. Desta forma, uma melhor avaliação é a endoscopia com o animal em exercício intenso, em esteira de alta velocidade (Lumsdem, 1995; Derksen, 2001). Devido ao alto custo da esteira existem apenas três destes equipamentos no Brasil, todos no Estado de São Paulo, o que evidencia a necessidade de novos métodos que sejam acessíveis economicamente, além de terem fácil aplicabilidade.

A captação de ruídos respiratórios através de sonda intranasal, posicionada na faringe, contendo microfone conectado a um gravador digital, permitirá a análise computadorizada da duração do ruído inspiratório, tempo inspiratório, duração do ruído expiratório, tempo expiratório, fator tempo inspiratório dividido pelo tempo expiratório e amplitude máxima dos decibéis no momento inspiratório e no momento expiratório, podendo possibilitar, através da padronização dos resultados, o diagnóstico da hemiplegia de laringe durante esforço físico intenso.

O presente estudo teve por objetivo avaliar a confiabilidade da análise dos ruídos respiratórios como técnica diagnóstica para a hemiplegia de laringe durante a atividade física intensa em condições reais de exercício em pista, bem como estabelecer comparações entre as alterações respiratórias entre cavalos saudáveis cavalos com hemiplegia de laringe experimentalmente induzida ou naturalmente adquirida.

\section{MATERIAL E MÉTODOS}

O experimento foi realizado nas dependências do Regimento Coronel Dulcídio, Polícia Militar do Paraná, e do Jockey Club do Paraná. Para a realização do estudo foram utilizados dez eqüinos, sete machos e três fêmeas, sendo sete animais da raça puro sangue inglês (PSI), um da raça quarto de milha, um mestiço PSI, e um da raça Brasileiro de Hipismo.

Os animais foram divididos em três grupos: Grupo controle, com animais saudáveis; Grupo HLI, com animais induzidos experimentalmente à hemiplegia de laringe graus IV e V; e Grupo HL, com animais naturalmente acometidos pela hemiplegia de laringe grau IV e $\mathrm{V}$.

Inicialmente foi realizada a avaliação endoscópica para confirmação da função respiratória normal nos grupos controle e $\mathrm{HLI}$, e para a confirmação da hemiplegia de graus IV ou V no grupo HL. O equipamento de captura de áudio consistiu em sonda flexível de $60 \mathrm{~cm}$ de comprimento e $08 \mathrm{~mm}$ de diâmetro, contendo um microfone de alta sensibilidade (aproximadamente 38 decibéis). O microfone foi ligado a um rádio-gravador digital, e este, acondicionado dentro de uma bolsa, junto ao corpo do cavaleiro. Antes de se realizar a técnica de gravação, no grupo HLI fez-se a indução da hemiplegia de laringe, 
injetando $5 \mathrm{ml}$ do anestésico local Lidocaína $2 \%$, sem vasoconstritor, próximo ao nervo laríngeo-recorrente esquerdo, dorso-caudalmente ao ponto de palpação da cartilagem cricóide. Para a confirmação da hemiplegia induzida foi realizada nova endoscopia cinco minutos após a aplicação do anestésico local.

Para gravação do áudio em todos os animais, introduziu-se a sonda na narina esquerda, até a porção cranial da nasofaringe, em seguida a sonda era dobrada e atada ao cabresto com esparadrapo. Assim que o rádio-gravador foi acionado, o cavalo iniciava cinco minutos de passo e cinco minutos de trote para aquecimento. Em seguida passava para o galope em alta velocidade, este sendo considerado o momento de esforço máximo do animal. Estes momentos foram anotados pelo observador, para posterior identificação da fração do áudio utilizada para análise gráfica.

O arquivo de áudio foi transferido para o computador, e selecionada a fração do som referente ao galope, utilizando-se o software PRAAT (versão 4.5.02) para a análise de áudio, onde após a identificação do segmento correspondente ao esforço máximo, os dados foram convertidos à freqüência média de 50 decibéis, para que houvesse padronização entre as amostras de áudio. Os dados obtidos de cinco movimentos respiratórios de cada amostra foram tabulados, e obteve-se a média para cada dado, individualmente, para os seguintes parâmetros: duração do ruído inspiratório, tempo inspiratório, duração do ruído expiratório, tempo expiratório, fator tempo inspiratório dividido pelo tempo expiratório e amplitude máxima dos decibéis no momento inspiratório e no momento expiratório.

Para a análise estatística dos dados, foi utilizada a análise de variância a um critério (ANOVA ONE - WAY), e caso encontradas diferenças significativas $(P<0,05)$, o teste de comparações múltiplas de Bonferroni foi aplicado. Todos os cálculos foram realizados utilizando 0 Software estatístico GraphPad Prism version 3.00 for Windows, San Diego Califórnia, EUA.

\section{RESULTADOS E DISCUSSÃO}

$\mathrm{Na}$ análise do ruído respiratório houve uma variação evidente de amplitude de decibéis inspiratórios nos testes realizados entre os cavalos dos grupos controle, HLI e HL. Observou-se no Grupo controle, que o gráfico do áudio correspondente ao galope demonstrou padrão respiratório simétrico, onde pode distinguir-se plenamente a diferença entre inspiração, com menor amplitude, e expiração, com maior amplitude (figura 1). No grupo HLI, o áudio apresentou padrão respiratório diferente do gráfico do grupo controle, evidenciado principalmente pelo aumento na amplitude média dos decibéis inspiratórios (figura 2). O gráfico do grupo HL obteve valores semelhantes ao Grupo HLI (figura 3).

Os valores de tempo inspiratório dividido pelo tempo expiratório apresentaram diferença significativa apenas nos animais com hemiplegia induzida, quando comparados aos valores do grupo controle, o que significa que estes cavalos passaram a apresentar um tempo inspiratório maior que o tempo expiratório, pela estenose dinâmica provocada, como também descrito por Tetens et al. (2001). Os animais naturalmente acometidos de hemiplegia, grupo HL, apresentaram valores muito próximos do Grupo HLI, entretanto, estes dados não foram significativos (figura 4). Provavelmente isto ocorreu devido a hemiplegia induzida provocar uma paralisia de grau $\mathrm{V}$, ou completa, enquanto nos animais naturalmente acometidos os graus podem se alterar durante 0 exercício em intensidade máxima, como foi demonstrado por Hammer et al. (1999).

Os valores dos decibéis inspiratórios dos grupos $\mathrm{HLI}$ e $\mathrm{HL}$ demonstraram 


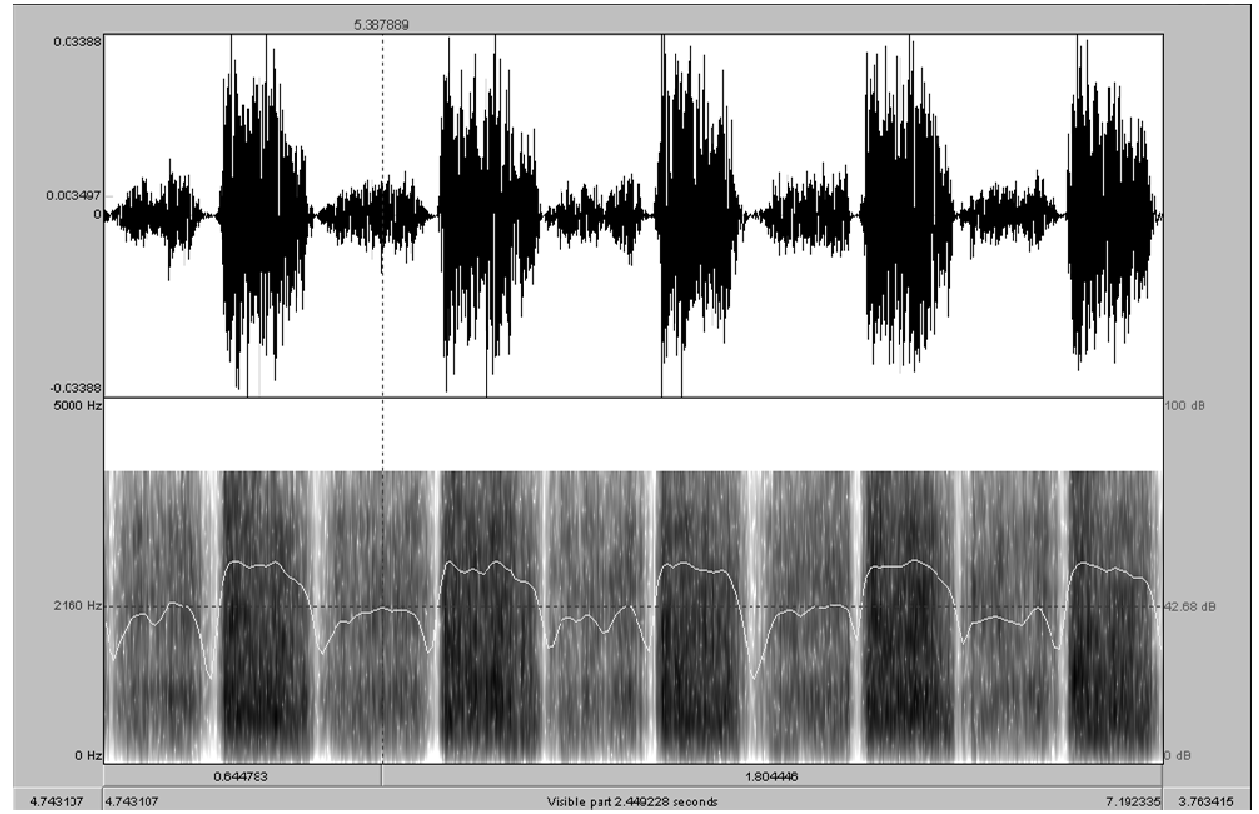

Figura 1- Gráfico do ruído respiratório de um cavalo do grupo controle demonstrando cinco movimentos respiratórios. A linha pontilhada vertical indica o ruído de uma inspiração.

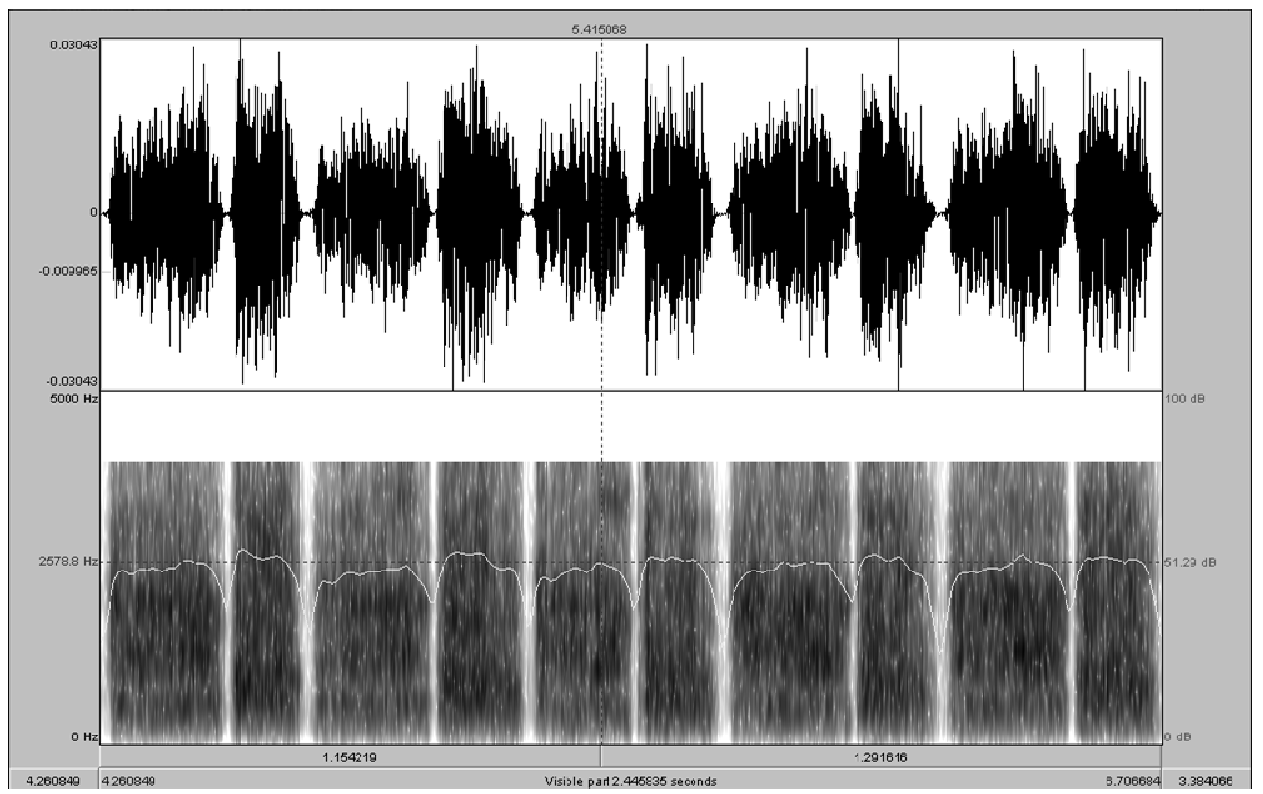

Figura 2 - Gráfico do ruído respiratório de um cavalo com hemiplegia de laringe experimentalmente induzida (grupo HLI), demonstrando cinco movimentos respiratórios. A linha pontilhada vertical indica o ruído de uma inspiração. 


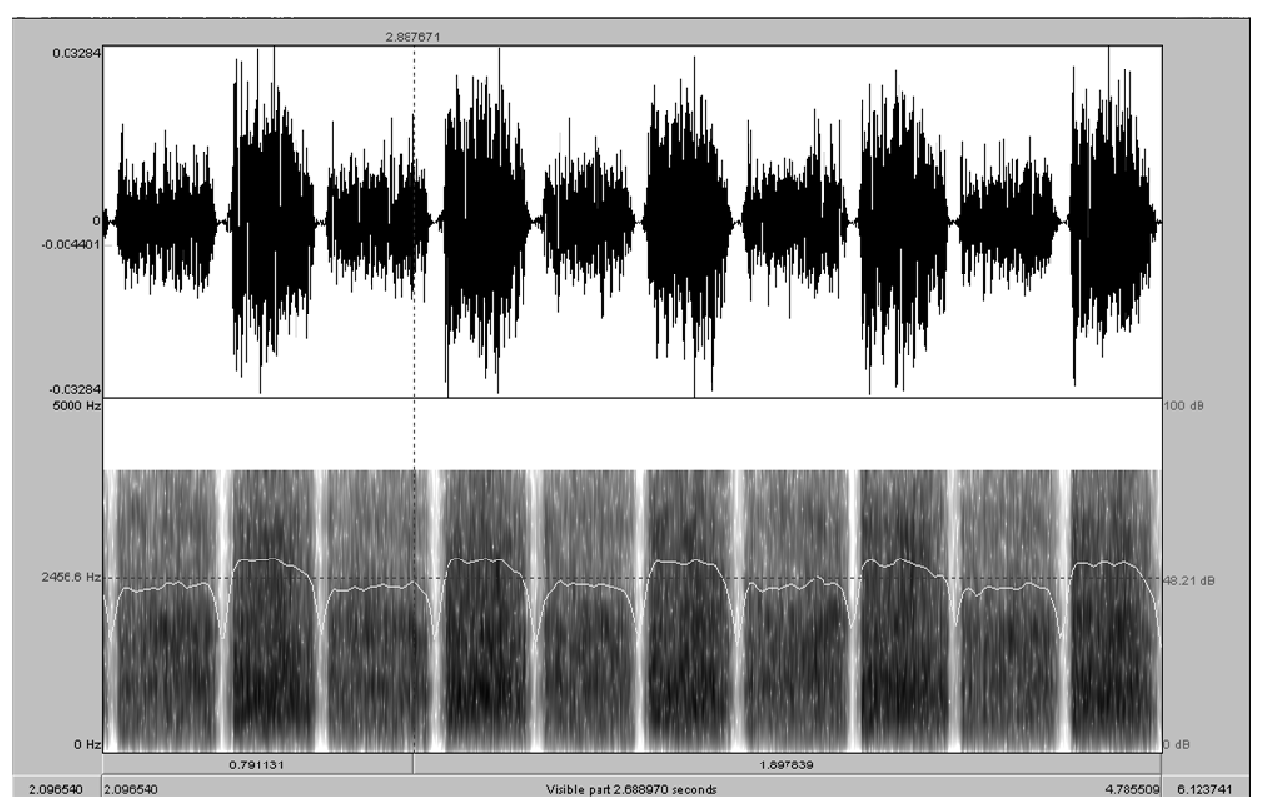

Figura 3 - Gráfico do ruído respiratório de um cavalo naturalmente acometido pela hemiplegia laringeana (grupo HL), demonstrando cinco movimentos respiratórios. A linha pontilhada vertical indica o ruído de uma inspiração.

diferença significativa quando comparados ao grupo controle (figura 5). Estes achados estão em conformidade com os obtidos por Derksen et al. (1999), que também encontraram os valores dos decibéis inspiratórios aumentados. Isto é explicado

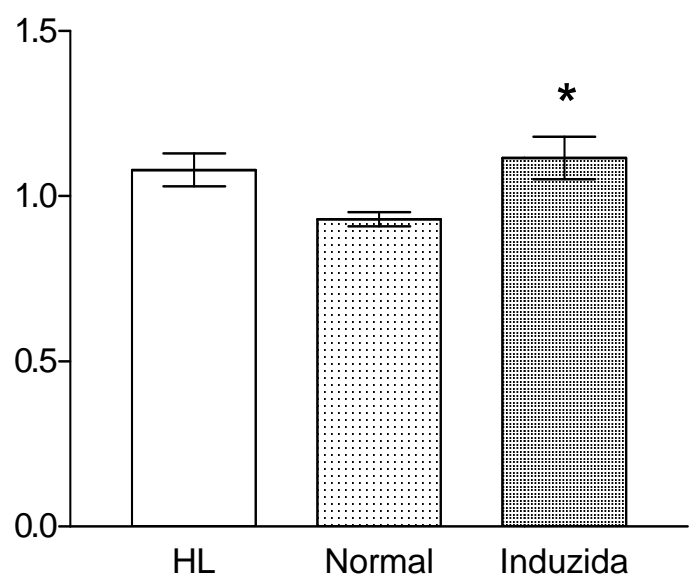

Figura 4 - Fator obtido pela divisão do tempo inspiratório pelo tempo expiratório, nos animais dos grupos de cavalos naturalmente acometidos pela hemiplegia laringeana $(\mathrm{HL})$, saudáveis (controle) e com hemiplegia de laringe experimentalmente induzida $(\mathrm{HLI}) .{ }^{*} \mathrm{P}<0,05$, em relação ao grupo normal. As barras indicam as médias \pm desvio padrão. pelo fato de a hemiplegia ser uma doença que altera principalmente a fase inspiratória da respiração, pois a não abdução das cartilagens aritenóides oferece resistência à passagem do ar, o que gera turbulência $\mathrm{e}$ um aumento nos ruídos. Os mesmos autores relataram que a fase expiratória não representou grande alteração porque a doença não tem relação com esta fase respiratória. Dados estes semelhantes aos obtidos no presente estudo.

A análise estatística dos valores, em milissegundos, obtidos da duração do ruído inspiratório, tempo inspiratório, duração do ruído expiratório, tempo expiratório, tempo da respiração e os decibéis máximos na expiração, não apresentaram diferenças significativas. Os valores respiratórios médios estão demonstrados na tabela 1 . Estas informações são semelhantes às obtidas por Derksen et al. (2001), com exceção dos valores do tempo inspiratório onde estes encontraram diferenças significativas, indicando que a inspiração tornou-se mais lenta. Este fato não foi confirmado neste estudo, talvez pelo fato de os animais galoparem com uma maior 
freqüência respiratória, conseqüentemente uma maior freqüência de passadas. Devendo ressaltar que a necessidade de exercitar os eqüinos a uma grande velocidade, ou seja, a um grande esforço físico para a obtenção de dados mais fidedignos já foi comprovada por Cable et al. (2002). Mais estudos são necessários para se determinar as alterações nos gráficos dos ruídos respiratórios, provocados pelas demais doenças que afetam as vias aéreas superiores, e que acabam interferindo de forma dinâmica na performance atlética dos cavalos.

A técnica de gravação de som por meio de microfone embutido em uma sonda posicionada na faringe aplicada neste estudo foi adaptada daquela utilizada inicialmente por Cable et al. (2002), que trabalharam com os animais em esteira e somente com hemiplegia induzida experimentalmente. Por sua vez Derksen et al. (2001) trabalharam com um microfone posicionado externamente, próximo a narina dos eqüinos, o que captava uma série de ruídos, principalmente os do contacto dos cascos com a esteira, que interferiram na qualidade do áudio obtido.

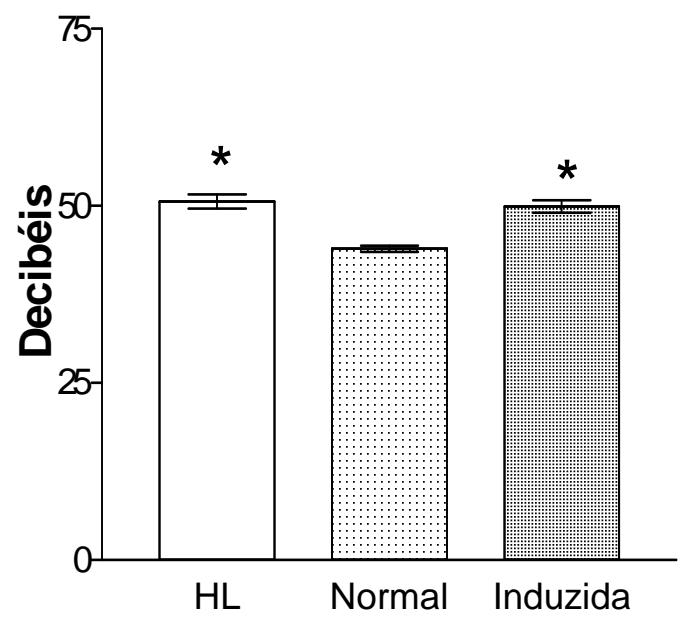

Figura 5 - Valores dos decibéis inspiratórios nos animais dos grupos de cavalos naturalmente acometidos pela hemiplegia laringeana (HL), saudáveis (controle) e com hemiplegia de laringe experimentalmente induzida (HLI). * $\mathrm{P}<0,05$, em relação ao grupo normal. As barras indicam as médias \pm desvio padrão.
A técnica associada ao exercício a campo, além de inédita, mostrou-se eficaz devido à facilidade de se conseguir o material para elaboração do equipamento, e também por este ser de custo menor quando comparado ao método de endoscopia em esteira, meio diagnóstico de eleição para afecções dinâmicas do trato respiratório superior.

Tabela 1 - Média dos valores respiratórios obtidos de cavalos saudáveis (controle), com hemiplegia induzida (HLI) e naturalmente acometidos (HL), as quais não demonstraram diferenças estatísticas significativas $(P<0,05)$.

\begin{tabular}{lccc}
\hline \multicolumn{1}{c}{ CRITÉRIO } & CONTROLE & HLI & HL \\
\hline $\begin{array}{l}\text { Duração do ruído } \\
\text { inspiratorio (ms) }\end{array}$ & 0,211 & 0,232 & 0,215 \\
$\begin{array}{l}\text { Tempo inspiratorio } \\
\text { (ms) }\end{array}$ & 0,237 & 0,251 & 0,241 \\
$\begin{array}{l}\text { Duração do ruido } \\
\text { expiratorio (ms) }\end{array}$ & 0,224 & 0,209 & 0,196 \\
$\begin{array}{l}\text { Tempo expiratorio } \\
\text { (ms) }\end{array}$ & 0,255 & 0,227 & 0,224 \\
$\begin{array}{l}\text { Tempo da } \\
\text { respiraçao (ms) }\end{array}$ & 0,491 & 0,479 & 0,464 \\
$\begin{array}{l}\text { Amplitude máxima } \\
\text { na expiração (dB) }\end{array}$ & 55,673 & 54,591 & 54,036 \\
\hline
\end{tabular}

O fato de o exame ser realizado no ambiente ao qual 0 animal esta familiarizado facilita a colaboração por parte do mesmo, pois se sabe que determinados animais relutam a subir em esteiras, 0 que acaba impedindo a realização do exame e um possível diagnóstico mais apurado (Ainsworth et al, 2005). A técnica empregada na captação do áudio em condições de campo, com o microfone posicionado na laringe é de fácil aplicação, bem tolerada pelos animais e permite a obtenção de amostras de áudio sem interferência de ruídos externos.

\section{CONCLUSÃO}

A amplitude máxima dos decibéis inspiratórios, sem modificações na amplitude máxima dos decibéis expiratórios, obtida com os eqüinos em atividade física intensa, mostrou ser 
significativamente diferente dos animais do Grupo controle, podendo desta forma ser utilizada no diagnóstico da hemiplegia de laringe em graus mais avançados, durante o exercício.

\section{Agradecimentos}

Programa de Iniciação Científica da Pontifícia Universidade Católica do Paraná.

\section{REFERÊNCIAS}

AINSWORTH, D. M.; MCGORUM, B. C.; VIEL, L.; ROBINSON, N. E.; DUCHARME, N. G.. Upper Airway Sound Analysis: Implications for Diagnosis and Surgical Treatment. 2005. Ithaca, NY. Disponível em <http://www.ivis.org/proceedings/ WEAS/2005/Derksen/chapter.asp?LA=1> Acesso em 10.out.2007.

CABLE, C.S., DUCHARME, N.G., HACKETT, R.P., ERB, H.N., MITCHELL, L.M., SODERHOLM, V. Sound signature for identification and quantification of upper airway disease in horses. American Journal Veterinary Research. v.63, p.707-1713, 2002.

DERKSEN, F.J.; HOLCOMBE, S.J.; HARTMANN, W.; ROBINSON, N.E.; STICK, J.A. Spectrogram analysis of respiratory sounds in exercising horses. In Proceedings: American Association of Equine Pratictioners. v.45, p. 314-315. 1999.

DERKSEN, F.J., HOLCOMBE, S.J., HARTMANN, W., ROBINSON, N.E., STICK,J.A. Spectrum analysis of respiratory sounds in exercising horses with experimentally induced laryngeal hemiplegia or dorsal displacement of soft palate. American Journal Veterinary Research. v.62, p.659-664. 2001.
HAMMER, E. J., TULLENERS, E. P., PARENTE, E. J. AND MARTIN, B. B. Videoendoscopic assessment of dynamic laryngeal function during exercise in horses with grade-III left laryngeal hemiparesis at rest: 26 cases (1992-1995). Journal. American Veterinary Medical Association. v.212, p.399-403. 1999.

HOLCOMBE, S. J. A Review of Upper Airway Anatomy and Physiology of the Horse. In: $8^{\text {th }}$ AAEP Annual Resort Simposium Rome, Italy. International Veterinary Information Service (www.ivis.org), Ithaca, New York, USA. 1-9. 2006.

LUMSDEN, J. M.; STICK, J. A.; CARON, J.; NICKELS, F. A.; BROWN, C. M.; GODBER, L. M.; DERKSEN, F. J. Upper airway function in performance horses: Videoendoscopy during highspeed treadmill exercise. Compendium Continum Education v.17, p.1134-1143. 1995.

MARTI, E.; OHNESORGE, B. Genetic Basis of Respiratory Disorders. 2002, Ithaca, NY. Disponível em <http://www.ivis.org/special_books/Lekeux/marti/IVI S.pdf> Acesso em 10.out.2007.

MORRIS, E. A.; SEEHERMAN, H. J. Evaluation of upper respiratory tract function during strenuous exercise in racehorses. Journal Americam Veterinary Medical Association. v.196, p.431-438. 1990.

ROBERTSON, J.T., DUCHARME, N.N. Disorders of the Pharynx and Larynx. 2005, Ithaca, NY. Disponível em <http://www.ivis.org/special_books/ Lekeux/robertson/chapter.asp?LA=1> Acesso em 10.out.2007.

TETENS, J., DERKSEN, F.J., HILLMANN, D.J. Idiopathic laryngeal hemiplegia. Compendium. v.23, n.1, p.85-93, 2001. 